a cure with almost dramatic suddenness. But it may perhaps be again pointed out that thrush (monilia) is not a common cause of a leucorrhoeal discharge.

Foreign Bodies. A forgotten and retained pessary which has long ago been inserted to relieve prolapse can cause profuse vaginal discharge, ulceration, and even carcinoma. A less neglected pessary can cause a lesser degree of discharge which the patient may describe as the "whites." Consequently some discussion of such a discharge may be permitted and it may here be urged that it is always desirable to instruct a patient who is wearing any pessary, be it a ring or a Hodge or other pessary and whether it be of rubber, vulcanite, metal or porcelain, to use a daily douche of some mild antiseptic solution or even normal saline. Admittedly this is not the teaching of everyone. Some gynaecologists do not like their pessary patients to use a douche and they maintain that these pessaries do not cause a discharge and that they do not indicate regular douching. But it has fallen to some of us to have the unpleasant experience of being required to change the pessary for a patient on this routine. The lesson is always salutary. After one such experience it will be that the doctor's invariable practice is to order douches for his pessary patients!

Of recent years one other foreign body has occasionally been the cause of a vaginal discharge which, though it can hardly be called leucorrhoea-for this discharge is purulent and stinking - should perhaps be mentioned here. The foreign body referred to is the internally worn vaginal tampon often used during menstruation instead of the sanitary towel: Whether it be that this method of female hygiene has always been potentially dangerous, or whether it be that the quality of the article usually sold has deteriorated during the present war, is neither here nor there. The fact is that several cases have occured recently in which, after pulling out something by means of the string incorporated in the tampon, and presumably dropping it unseen down a lavatory, part or possibly the whole of the cotton wool plug has remained in the vagina and caused a blockage. Above this, menstrual fluid, cervical discharge, etc., has accumulated and its subsequent infection is inevitable. The result is that the upper part of the vagina has become to all intents and purposes an abscess cavity. The stinking purulent fluid seeping through or leaking past the plug has led to the appearance of a most offensive discharge, which, as a rule rapidly and spontaneously clears up, after removal of the obstruction to the vaginal outflow. But it can well be imagined that more serious infections might arise and a persisting cervical "erosion" appears to have been a fairly common sequel. This in its turn would lead to a persistent discharge to which the term leucorrhoea can more correctly be applied.

REFERENCE

(I) BOURNE, A. W., BOND, L. T., and McGARRITY, K. A., Brit. Med. Jour. 1937. 1, 116.

\title{
SOME RECENT ADVANCES IN THE APPLICATION OF ANTENATAL CARE
}

\author{
By JOSEPHINE BARNES, M.A., D.M., M.R.C.P., F.R.C.S., M.R.C.O.G. \\ (First Assistant in the Obstetric Unit and Obstetric Registrar, University \\ College Hospital)
}

The expectant mother is one of the most valuable assets of the community, more especially since the recent alarming fall in the birth-rate. 'It is, however, only in recent years that antenatal care has been recognised as of importance, either to the mother, or to the community as a whole. The establishment of hospital beds for the treatment of disorders of pregnancy has only occurred within the twentieth century, but in the space of a little over forty years many advances have been made, all of which are impossible to deal with in the scope of an article of this nature. There is much to criticise and many advances to be made in antenatal care. Here, it is proposed to discuss critically some of the aspects of the subject in which advance has been made recently, and in which further advance can be expected in the future. 


\section{Diet in Pregnancy.}

With the recent advance of biochemistry, and especially since the discovery of the vitamins, the importance of sound nutrition has been increasingly emphasised. But malnutrition is a word that is often used loosely, without reference to its real meaning, namely, that it is the quality, rather than the quantity, of food consumed that is important. The requirements of the expectant and nursing mother were laid down recently, and an adequate diet has been suggested by Mellanby (I933). He showed that no increase in the total caloric intake is necessary in pregnancy, but it is of paramount importance to have an increased intake of certain vital foodstuffs, namely, animal protein, vitamins, certain mineral salts, and iodine.

Mellanby stated that the basic diet in pregnancy should contain the following:-

2 pints of milk daily.

I or 2 substantial servings of green vegetábles.

I or 2 eggs or egg yolks daily.

An apple, orange, or other fresh fruit daily.

Sea fish twice, or oftener, a week.

Calves' liver once a week.

Cod-liver oil, two teaspoonfuls daily, if it can be taken.

The rest of the diet can be made up to the desires of the patient.

Such a diet was beyond the reach of the poorer mother before the war, and in spite of commendable efforts to improve the diet of expectant and nursing mothers, by providing extra milk and eggs, there is a danger that these patients may be mal-nourished in war-time. Under the present rationing system, a deficiency of animal protein cannot be remedied, but deficiencies of other vital substances can be met by giving them as supplements to the diet. Hamilton and Payling Wright (I942) showed that iron deficiency anaemia is common among pregnant women, and by comparison with other published figures, concluded that women of the artisan class are more anaemic than before the war. They recommend the administration of iron as a routine during pregnancy. Calcium deficiency can be met by the administration of a suitable preparation, such as the "Tabletta Calcii cum Vitamino D". of the National War Formulary. Vitamin deficiencies can be met by advice on diet and the administration of suitable concentrates. Deficiency of vitamin A seems to be especially liable to occur. Mellanby and Green (I928) showed that the morbidity rate of patients who received vitamins A and D was appreciably lower than in a control group, in whom no such addition was made. This suggests that a deficiency of vitamin A may predispose to puerperal infection.

The People's League of Health set up a committee to investigate the nutrition of expectant mothers, and the interim report (I942) of this committee has recently been published. The general conclusion on diet was that an improvement had occurred recently, probably on account of advice given at antenatal clinics. Deficiencies of iron, calcium and vitamins $\mathrm{A}$ and $\mathrm{C}$ were especially noted. A total of 5,02I expectant mothers were divided into two groups, one of which received dietary supplements of vitamins and mineral salts. It was found that the incidence of toxaemia of pregnancy was significantly lower in the treated group than in the control group, and that in the treated group there was a greater likelihood of the mother carrying the pregnancy to term. There seemed to be no effect on the character or duration of labour or on the incidence of puerperal pyrexia and puerperal sepsis. These results thus differ from those of Mellanby and Green already quoted.

The educational aspect of diet is important, since it is useless for experts to draw up ideal dietaries if the practical aspect is not brought home to the patient. Malnutrition arises from two main causes-bad economic conditions and ignorance. The solution of the latter is to educate the expectant mother by means of lectures and demonstrations on diet and the proper preparation of food. Such lectures might well be given in all antenatal clinics.

\section{Contracted Pelvis and Disproportion.}

The most recent advances in the study of the obstetric pelvis have come from the use of radiography. As a result of this method of investigation, the variations in the shape of the pelvis have been studied and classified, and a much more accurate estimation of the pelvic capacity can be made than is possible by clinical methods alone. This is not to say that clinical methods, especially the measurement of the true conjugate by the Muller-Munro Kerr manoeuvre, are not of the greatest value. The value of X-ray pelvimetry lies in the help it is able to give 
in many cases where the outlook for labour, from the clinical estimation alone, is in doubt. A gross degree of contracted pelvis is easily diagnosed, but this condition, at least in the south of England, seems to be becoming more and more uncommon. This is probably.due to the virtual disappearance of rickets in this area. A minor degree of disproportion is still fairly common, and is in some ways a greater danger to the mother and foetus, since the difficulty may not be anticipated until labour is far advanced. Until recently, stress has been laid mainly upon contraction at the pelvic inlet, but it is now recognised that difficulty may arise at any level in the pelvis, though especially at the inlet and outlet. Whitridge Williams, Thoms, Caldwell and Moloy, Ince and Young, Munro Kerr and Moir have all stressed the importance of the funnel pelvis. In this type of pelvis delay occurs at the outlet, or sometimes in the pelvic cavity, and may necessitate a forceps delivery which is often extremely difficult. It may lead also to severe laceration of the soft parts, to delay in the dilation of the cervix, and may predispose to the production of a contraction ring. Contraction of the pelvic outlet can often be recognised by clinical methods; the digital method described by Moir (I94I) is the most accurate and should be carried out as a routine in all antenatal patients. This can be done at the first clinical examination or in the later months of pregnancy.

$\mathrm{X}$-ray pelvimetry is a method of investigation that is still in the process of development. In this country, the method is available to comparatively few antenatal patients and progress in its application has been retarded. As Moir (I94I) pointed out in a survey of pelvic contraction, this lack of progress had been due largely to failure of co-operation between obstetricians and radiologists. The acme of perfection is for the $\mathrm{X}$-ray plant to be in the maternity hospital, so that X-rays can be taken not only during pregnancy, but also in the course of labour; so often, however, the patient has to go some distance to have radiographs taken and a report has to be sent to the obstetrician who loses the advantage of personal co-operation with the radiologist. A careful technique and accurate measurement are two other essentials which are not always realised, and bad or inaccurate X-ray pelvimetry is misleading and dangerous. A good X-ray of the pelvis, however, can be of inestimable value, and in Moir's article he points out that a lateral $\mathrm{X}$-ray taken with the patient standing may give all the information required, at a very low cost.

Foetal cephalometry by means of $\mathrm{X}$-rays is another method of investigation that has come into use of recent years. Accurate measurement of the size of the foetal head in utero is not easy, and even if the size of the head is known it is still difficult to prophesy how much the head will mould during labour. However, this may be a valuable.method of investigation, especially in the case of breech presentation, in which it is impossible to determine by clinical methods whether the head will pass the pelvic brim or not.

In summary it may be stated that $\mathrm{X}$-ray pelvimetry is likely to become increasingly important, and its use should certainly.be extended. The following types of case should have X-ray pelvimetry performed:-

I. Primigravidae in whom contracted pelvis is suspected, either on account of short stature or evidence of past rickets.

2. Primigravidae in whom, in the absence of an obvious obstruction such as a pelvic tumour, the head is not engaged in the pelvis by the thirty-seventh week of pregnancy.

3. Any patient with a bad obstetric history, i.e. the history of a prolonged labour or difficult forceps delivery.

4. Cases of breech presentation where external version cannot be performed. In these cases, foetal cephalometry should also be performed. In a primigravida, where there is even slight contraction of the pelvis, Caesarean section may be the best treatment.

Dippel (I939) states that X-ray pelvimetry is desirable when the diagonal conjugate by clinical measurement is less than $\mathrm{II} \cdot 5 \mathrm{~cm}$. (4.7 inches).

In the management of contracted pelvis, three methods of treatment are availableCaesarean section, trial labour, and induction of premature labour. Caesarean section should be performed in cases of severe disproportion and in the elderly primigravida.

The modern tendency has been to abandon induction of labour in favour of trial labour, chiefly on the grounds that many inductions formerly performed were unnecessary and carried a great risk of subsequent sepsis. Barnett (I942) in an analysis of cases of induction and trial labour carried out at University College Hospital, showed that the induction cases carried a lower Caesarean section rate, a lower foetal and neo-natal mortality, and a lower morbidity rate. It seems that as X-ray pelvimetry becomes more universally adopted, we shall be able to predict 
the course of labour with more certainty. Then, as trial labours or inductions would become less necessary, reconsideration of the relative merits of these two methods of treatment may be required.

\section{Placenta Praevia.}

This is a condition of such danger to mother and child that treatment should never be carried out except in an institution where facilities are available for blood transfusion and for such operative treatment as may become necessary. A small haemorrhage may be a warning of severe bleeding in the future, and any patient in whom such antepartum haemorrhage occurs should be transferred immediately to a maternity hospital or similar institution, without any attempt either to examine per vaginam or to pack the vagina. As regards the treatment of the patient when she is in hospital there is a difference of opinion. Moir (I942) believes that the danger of further severe haemorrhage is so great that an examination to exclude a major degree of placenta praevia should be carried out at once. Browne (I942) prefers to treat such cases expectantly if the bleeding is not severe and does not recur more than twice. The patient must, however, be kept under observation in hospital until delivery has taken place. In major degrees of placenta praevia, that is, where the placenta covers the internal os, Caesarean section has been shown to give the lowest maternal and foetal mortality, and it is generally agreed that this is the best treatment for these cases.

Attempts have been made to determine the position of the placenta by radiography. Dippel and Brown (I940) claimed to be able to visualise the placenta by a soft tissue radiography in 90 per cent of cases. Lloyd and Samuel (I94I) used tomography for the same purpose. Other methods have been used, such as the injection of an opaque substance (sodium iodide) into the bladder (Ude, Weum, and Urner) or of some similar substance into the amniotic cavity. For the latter, Uroselectan or Perabrodil have been used, but here there is a considerable risk of premature labour and foetal death.

A reliable method of determining the placental site would be a great advance in the management of cases of placenta praevia, but experience so far seems to show that the ideal method remains to be found.

\section{Pyelitis in Pregnancy.}

Advances have been made recently in the treatment of this condition. The sulphanilamide group of drugs has proved particularly successful. Kenny, Johnston, Haebler and Miles (I937) found that sulphanilamide (Prontosil album, Bayer) in doses of 0.5 gramme three times daily caused the urine to become sterile in five to seven days. Mandelic acid, or its sodium calcium or ammonium salt, also give good results, though it is unwise to give it during the acute phase which should be treated with alkalis until the symptoms are relieved.

The remote prognosis of pyelitis of pregnancy has hitherto been very unfavourable. Dodds (I932) in a series of cases followed up after delivery, showed that only 49 per cent were completely cured, while 35 per cent developed a chronic pyelitis. It is to be hoped that the newer methods will improve the results in what has so far proved so often to be an intractable condition.

\section{The Toxaemias of Late Pregnancy.}

In no other field in the whole of medicine has there been more research in recent years than in that of the toxaemias of pregnancy. Much remains to be solved, and the most difficult problem is that of the aetiology of eclampsia. Numerous theories have been proposed, but none has so far proved adequate to explain all the facts. In two aspects of the subject, progress appears to have been made recently, namely the classification of these disorders and their prognosis. Here it is proposed to discuss only these two aspects.

Classification of the toxaemias in late pregnancy. In the last ten years, much has been done to identify the various types of toxaemia. Formerly, it was the custom to speak of "albuminuria of pregnancy" or "pregnancy kidney." All the recent work has emphasised the relatively great importance of the vascular system, while the renal lesions are now recognised to be secondary.

Browne (I942) proposes a simple and convenient classification:-

I. Pre-eclamptic toxaemia and eclampsia.

2. Chronic glomerular nephritis and pregnancy. 
3. Essential hypertension in pregnancy.

4. Acute yellow atrophy of the liver.

Pre-eclamptic toxaemia is defined by Browne as "a condition occurring in pregnant women characterised chiefly by a rise of blood pressure, oedema and albumin in the urine, and often ending in convulsions." In this condition the signs of toxaemia do not appear before the twentieth week of pregnancy.

Chronic nephritis is rare in association with pregnancy. In a series of cases studied by Browne and Dodds (I939) chronic nephritis accounted for only seventeen out of four hundred patients who had suffered from pregnancy toxaemia and who had been followed up in order to assess the remote prognosis. They point out that the diagnosis may be extremely difficult. They rely mainly on a previous history of acute nephritis and to a less-extent on persistent albuminuria or unsatisfactory renal function tests.

Hypertensive toxaemia. Here the patient is known to have had hypertension before pregnancy or a raised blood pressure has been found before the twentieth week of pregnancy. There is often a family history of hypertension and its associated conditions.

Acute yellow atrophy of the liver. Several conditions were formerly included under this heading. Sheehan (I940) has shown that four separate groups of such cases can be distinguished.

I. True acute yellow atrophy. This is rare in pregnancy.

2. Obstetric acute yellow atrophy. This was found in six out of four hundred post mortems in obstetric cases. The onset was usually about the thirty-sixth to fortieth week. The foetus was stillborn, and the mother usually died within three to four days of delivery.

3. Delayed chloroform poisoning. This was found in fourteen of the four hundred post mortems. Patients become more sensitive to chloroform in prolonged labour, vomiting or acidosis, physical exhaustion, nitrogen retention and uterine sepsis. Chloroform, therefore, should never be used in the presence of any of these conditions.

4. Liver injury due to other poisons, such as mercury, arsenic, phosphorus and cincophen or its derivatives.

This new conception of the toxaemias shows how important the blood pressure is in diagnosis. The term "albuminuria of pregnancy" should be abandoned as it suggests that in the absence of albumin all is well with the patient. This is far from the truth, as severe toxaemia and even eclampsia may occur without the appearance of albumin in the urine. Regular estimation of the blood pressure is an essential part of antenatal care. There is still some controversy about the level of blood pressure which should be considered pathological. At University College Hospital, a blood pressure above $130 / 70$ is considered to be abnormally raised. Some consider this standard to be too exacting. Recent work from America, however, suggests that the accepted standards of "normal blood pressure" should be revised. Robinson and Brucer (I939) published an extremely important paper which should be read by all interested in the subject. It is impossible to give more than the barest outline of their conclusions here. Their results were based on a study of 10,883 persons, and a five-year survey of 500 cases. They show that hypertension is an extremely common condition, and indeed 40 per cent of the adult population is actually or incipiently hypertensive. The onset of the disease occurs in early adult life, and the blood pressure tends to rise progressively with advancing years, whereas normal blood pressure does not rise with age. They state that the normal systolic blood pressure is 90 to $\mathrm{r} 20 \mathrm{~mm}$. of mercury, and the normal diastolic blood pressure 60 to $80 \mathrm{~mm}$. of mercury. A blood pressure of over $120 / 80$ is pathological, and once the pressure is established at above this level, it seldom, if ever, returns to normal.

The prognosis of the toxaemias of late pregnancy. The remote prognosis of the toxaemias of late pregnancy was studied by Browne and Dodds (I939). In pre-eclamptic toxaemia the residual lesion was found to be invariably hypertension, and this was found in $50 \cdot 8$ per cent of their cases who were followed up for varying periods up to twelve years. In eclampsia the residual lesion was also hypertension, and was found in 60.8 per cent of the cases. This suggests that toxaemia of pregnancy tends to cause hypertension, but recent work suggests that this is apparently not the case. Isenhour, Kuder and Dill (I942) published a study of the blood pressure in 900 parous women and compared it with that of 900 nulliparous women. They found no significant difference between the two groups, even at different age levels. The systolic blood pressure was slightly higher in parous women than in nulliparous, but this could be accounted for by the fact that the body weight in parous women tends to be above that in nulliparous women, and they were able to show a close correlation between body weight and 
systolic blood pressure. They showed that patients who have toxaemia of pregnancy and subsequently develop hypertension would have done so in any case if they had not been pregnant.

These results are obviously of the greatest importance since they indicate that a severe toxaemia does not do the mother any permanent harm, and thus it is not necessary, on account of the mother, to terminate pregnancy when a high blood pressure is found. Termination may be necessary if there is impending eclampsia or in the interests of the foetus. Browne and Dodds found that in hypertensive toxaemia the majority of patients seemed to pass through their pregnancies without any deterioration in their condition, though in a subsequent twelveyear period 9.2 per cent of them had died. The same authors published in I 942 a study of 239 pregnancies in hypertensive patients. An important conclusion was that the prognosis for the foetus depended on the height of the blood pressure at the start of pregnancy. If this was I50/IOO or over, 68 per cent of the foetuses were lost, whereas the foetal mortality over the whole series was $\mathrm{I} 6 \cdot 2$ per cent.

Browne and Dodds (I935) in their study of 400 cases of pregnancy toxaemia observed II4 cases of recurrent toxaemia in 278 pregnancies. They found that 60 per cent of these patients had hypertension between their pregnancies, while the remainder were considered to be potential hypertensives. In pregnancy with chronic nephritis, however, the prognosis for the mother was bad, and in the following twelve years of the survey 29.4 per cent of the chronic nephritic patients had died. There is, of course, nothing to indicate what would have happened to these patients if they had not become pregnant.

In summary, the present position as regards the prognosis of the toxaemias of late pregnancy seems to be as follows: Pre-eclamptic toxaemia and eclampsia may be followed by chronic hypertension. This condition does not occur in all cases, and is not caused by the toxaemia, but would have occurred even if the patient had not been pregnant. Pregnancy does not cause permanent harm to the hypertensive mother, but here the prognosis for the foetus is poor if the blood pressure at the start of pregnancy exceeds $150 / 100$. In chronic nephritis and pregnancy the prognosis for the mother is poor.

\section{Conclusions.}

An attempt has been made here to outline some of the advances which have taken place in recent years in the application of antenatal care. The subject is now so large that it has been impossible to give more than the merest indication of the possible lines of progress. There is still room for improvement. Many criticisms can be directed against antenatal care as practised in this country to-day. Only too often so-called antenatal -care leads to unnecessary interference with the normal course of pregnancy, and especially to unnecessary Caesarean section. It has already been shown that with the increasing use of $\mathrm{X}$-ray pelvimetry we may hope to predict the course of labour with greater accuracy than has hitherto been possible, and this should help to diminish unjustifiable interference in cases where disproportion is suspected.

Another criticism is that a large proportion of antenatal work is done by officers of the Local Authorities at antenatal clinics often unconnected with maternity hospitals. These medical officers are responsible for the patient only during the antenatal period, and do not háve the opportunity of following the case through the confinement and puerperium. Not only does this make the work a matter of dull routine, but the patient loses the continuity of treatment which is so desirable in pregnancy. This could be remedied by providing more hospital beds for maternity patients. It is to be hoped that this will be an early step in postwar reconstruction.

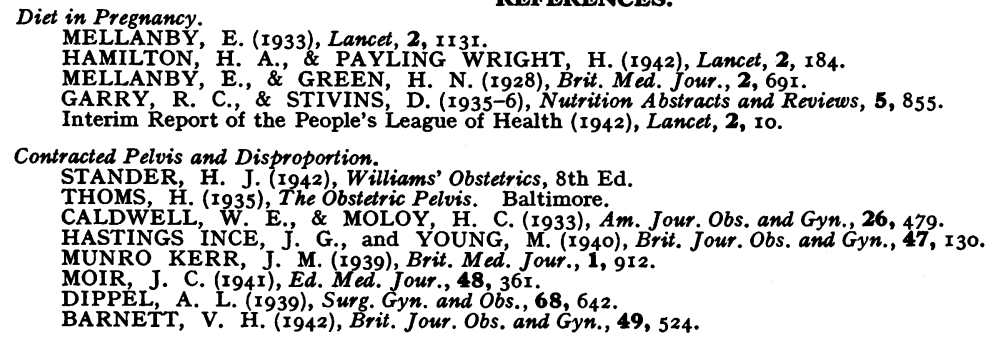




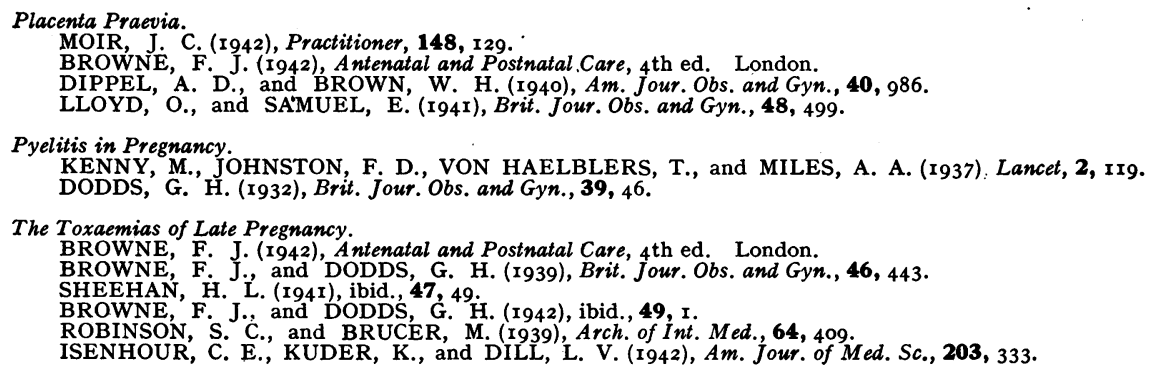

\title{
Practicalities
}

(Vol. I. No. 6)

\section{THE ELECTROCARDIOGRAPH IN DIAGNOSIS OF CARDIO-VASCULAR DISEASES}

\author{
By F. CROXON DELLER, M.D., D.A.
}

Before discussing some of the abnormal electrocardiograms which will be found in disease it would be well to consider the principles underlying the electrocardiograph.

If a strip of muscle is isolated and connected at each end to the poles of a galvanometer, the application of a single electrical stimulus to one end of that strip will cause a contraction at the point stimulated. This area immediately becomes electrically negative in relation to the unstimulated end, and an electrical current flows from positive to negative, causing a deflection, say, to the left, of the galvanometer needle. The contraction wave then passes along the muscle strip so that when it arrives at the centre of the muscle strip the negative charge is in the middle, whilst the two ends hold a positive charge; when this occurs no current flows through the galvanometer. The needle of the galvanometer will therefore come to rest again in the central position. The contraction wave continues, and on arrival at the end opposite to that originally stimulated, this becomes negatively charged and the direction of flow of the electrical current is reversed, the galvanometer swinging over to the right. These deflections can be recorded by means of the reflection of a beam of light from a mirror attached to the needle of the galvanometer; it can be arranged so that a flow of current in one direction will cause a deflection of the beam of light, say, in an upward direction, whereas a flow of current in the opposite direction will cause a deflection in a downward direction. Once, therefore, standardisation of the flow of current has been obtained, it will be possible to collate the various deflections produced in a strip of muscle, and to make deductions from these changes of any abnormalities that may be present in the muscular contraction.

Normally, in clinical electrocardiography three leads are used, by placing the electrodes upon the left wrist, the right wrist and the left leg just above the ankle. From the electrical point of view these leads are at an infinite distance from the heart muscle and measure the changes in electrical potential which are occurring due to the cardiac contractions. These leads record the changes produced firstly across the base of the heart, secondly along the right border, and thirdly along the left border of the heart, and are known as Lead I, taken from left arm to right arm, Lead II, from right arm to left leg, and Lead III, from left leg to left arm. Further research into electrocardiographic changes showed that it was often possible to detect slight changes in electrical potential not recorded by these classical leads, by moving one or two of the leads and placing them much nearer to the heart. This is known as Lead IV, or the chest lead, and its significance will be discussed in relation to coronary diseases.

The normal standardisation employed is that I millivolt change of potential will cause a deflection either upward or downward from the isoelectric line of I centimetre in height; the isoelectric line being that line produced upon the record when no changes of electrical potential are occurring. 